\title{
Seasonal rotation features of wind vectors and application to evaluate monsoon simulations in AMIP models
}

\author{
Li Zhang $\cdot$ Jianping Li
}

Published online: 19 December 2007

(C) Springer-Verlag 2007

\section{Erratum to: Clim Dyn \\ DOI: 10.1007/s00382-007-0327-9}

In the original article some keywords were unfortunately incorrect. The corrections are as follows:

Section 2.2, the second paragraph:

Lines 11, 13, and 14: The three words, "counterclockwise" should be "clockwise".

Lines 18 and 20: The two words, "clockwise" should be "counter-clockwise".

\subsection{Directed angle and its difference with absolute} angle

In traditional studies, the angle between two prevailing surface wind vectors is calculated as follows:

The online version of the original article can be found under doi:10.1007/s00382-007-0327-9.

\section{Zhang - J. Li}

State Key Laboratory of Numerical Modeling for Atmospheric Sciences and Geophysical Fluid Dynamics (LASG),

Institute of Atmosphere Physics, Chinese Academy of Sciences, Beijing 100029, China

L. Zhang

Graduate University of Chinese Academy of Sciences,

Beijing 100049, China

J. Li (\)

LASG, Institute of Atmospheric Physics,

Chinese Academy of Sciences, P.O. Box 9804,

Beijing 100029, China

e-mail: ljp@lasg.iap.ac.cn
$\beta_{j}=\beta\left(\mathbf{V}_{j}, \mathbf{V}_{\mathrm{R}}\right)=\arccos \left(\frac{\left(\mathbf{V}_{j}, \mathbf{V}_{\mathrm{R}}\right)}{\left|\mathbf{V}_{j}\right|\left|\mathbf{V}_{\mathrm{R}}\right|}\right), \quad(j=1, \ldots, 365)$,

where $\mathbf{V}_{j}$ is the daily wind vector, and $\mathbf{V}_{\mathrm{R}}$ is the reference wind vector at the same position. In this study, we choose the January climatological wind vector (one could, however, choose any month) as the reference wind vector. The norm $\left(\mathbf{V}_{j}, \mathbf{V}_{\mathrm{R}}\right)$ means vector product, and $|\cdot|$ denotes the module of the wind vector. Thus $\boldsymbol{\beta}_{j}$ essentially measures the contrast of the wind vector angles between a specific day and the corresponding winter. Obviously, $0 \leq \beta_{j} \leq$ $180^{\circ}$, that is, $\boldsymbol{\beta}_{j}$ is always positive, so here it is named the absolute angle.

The absolute angle between prevailing surface wind vectors of summer and winter was used to describe and study monsoons in previous studies (e.g. Khromov 1978; Li and Zeng 2000). However, the definition of absolute angle does not include the rotation direction of the wind vector. Actually, the variations in wind direction during seasonal evolution are not consistent in different areas. Figure 1a indicates the scattered representation of normalized wind vectors at $\left(10^{\circ} \mathrm{N}, 55^{\circ} \mathrm{E}\right)$. The dots denote the directions of daily wind vectors. It is obvious that the wind vector rotates clockwise from its winter state (Jan 1-May 9) to its summer state (May 16-Oct 8). Then, it continuously rotates clockwise and returns to its initial state. During the whole process, the wind vector rotates clockwise by almost $360^{\circ}$. However, the rotation direction of the wind vector at $\left(15^{\circ} \mathrm{N}, 45^{\circ} \mathrm{E}\right.$; see Fig. 1b) is quite different. Though the rotation amplitude is as large as $360^{\circ}$, the rotation direction is completely reverse. It rotates counterclockwise from its winter state (Jan 1-Jun 1) to its summer state (Jun 10-Sep 8), and continuously rotates counterclockwise to its initial state. This suggests that the rotation 
amplitude of the wind vector in an annual cycle is not always between $0^{\circ}$ and $180^{\circ}$ as the absolute angle defined. Meanwhile, the absolute angle is not able to detect and describe the rotation direction of the wind vector. Therefore, here, we introduce the concept of directed angle, which is a signed measure of the angle. It has the ability to distinguish different rotation directions and amplitudes to describe the whole process of wind vector rotation. In this study, we define the counter-clockwise (clockwise) rotation as the positive (negative) value of the angle. Considering the gradual change of climatological daily wind vectors, we adopt a day-to-day recursion method to calculate the daily directed angle $\alpha$ as follows:

$\alpha_{1}=\alpha\left(\mathbf{V}_{1}, \mathbf{V}_{\mathrm{R}}\right)=\delta \beta_{1}$,
$\alpha_{i}=\alpha\left(\mathbf{V}_{i}, \mathbf{V}_{\mathrm{R}}\right)=\alpha_{i-1}+\delta \beta\left(\mathbf{V}_{i}, \mathbf{V}_{i-1}\right), \quad(i=2, \ldots, 365)$,

where $\delta$ is the signed function to determine the rotation direction and $\mathbf{V}_{\mathrm{R}}$ is the reference wind vector (we choose the January climatological wind vector). If $\mathbf{V}_{\mathbf{i}}\left(\mathbf{V}_{1}\right)$ is in the clockwise (counter-clockwise) direction of $\mathbf{V}_{i-1}\left(\mathbf{V}_{\mathrm{R}}\right)$, then $\delta=-1(1) . \beta$ is the absolute angle between the specific day and the day before. From the calculated formulation, it can be seen that the concept of directed angle contains the rotation direction of the wind vector, which is much more visual and objective than the absolute angle to investigate variations in wind direction. 